\title{
Custo da Doença em Pacientes com Carcinoma Mamário Tratados com Tamoxifeno
}

doi: https://doi.org/10.32635/2176-9745.RBC.2019v65n2.372

\author{
Cost of the Disease in Patients with Breast Cancer Treated with Tamoxifen \\ Costo de la Enfermedad en Pacientes com Carcinoma Mamário Tratados com Tamoxifeno
}

\author{
Mariangela Adriane Seroiska'; Luana Lenzi ${ }^{2}$; Astrid Wiens ${ }^{3}$
}

Resumo

Introduçáo: A estimativa do Instituto Nacional de Câncer José Alencar Gomes da Silva para o Brasil, para cada ano do biênio 20182019, foi de 59.700 novos casos de câncer de mama, com um risco estimado de 56,33 casos a cada 100 mil mulheres. Em 2014, os gastos públicos com atenção oncológica foram de aproximadamente R\$ 2,5 bilhóes. Objetivo: Avaliar o impacto dos custos diretos médicos em pacientes com carcinoma mamário tratados com tamoxifeno pelo Sistema Único de Saúde. Método: Estudo exploratório de custo da doença, quantitativo, retrospectivo, com caráter de prevalência e de abordagem bottom-up. A coleta dos dados foi realizada no ambulatório de Oncologia do Hospital São Vicente em Curitiba, Paraná. Resultados: A média do custo do tratamento dos pacientes foi R \$ 14.497,70 em tratamento neoadjuvante ou adjuvante e de R \$ 9.108,60 em tratamento paliativo. Em relaçáo a essas variáveis, o custo do tamoxifeno foi o que mais impactou em relação ao custo total do tratamento, representando mais de $80 \%$ deste valor. A média do custo anual gasto com tamoxifeno por paciente foi de R\$1.947,60. Conclusáo: O custo médio do tratamento demonstrou ser alto em relaçáo à média salarial dos brasileiros de R 2.110.00 (IBGE-2017). Os custos levantados neste estudo podem auxiliar os gestores de saúde pública em estratégias para racionalização dos gastos, otimização do capital e manutençáo do atendimento à população.

Palavras-chave: Neoplasias da Mama/economia; Tamoxifeno; Sistema Único de Saúde.

\begin{abstract}
Introduction: The estimate of the National Cancer Institute José Alencar Gomes da Silva, for each year of the 2018-2019 biennium in Brazil, was 59,700 new cases of breast cancer, with an estimated risk of 56.33 cases per 100,000 women. In 2014, public expenditures on cancer care were approximately $\mathrm{R} \$ 2.5$ billion. Objective: To evaluate the impact of direct medical costs on breast cancer patients treated with tamoxifen using the Unified Health System. Method: Prospective, quantitative, retrospective, cost-of-disease study with prevalence and bottom-up approach. Data collection was performed at the Oncology Outpatient Clinic of the Hospital São Vicente in Curitiba, Paraná. Results: The mean cost of the treatment was $\mathrm{R} \$ 14,497.70$ for neoadjuvant or adjuvant treatment and $\mathrm{R} \$$ $9,108.60$ for palliative treatment. In relation to these variables, the cost of tamoxifen was the one that most impacted, in relation to the total cost of the treatment, representing more than $80 \%$ of this value. The mean annual cost of tamoxifen per patient was R $\$ 1,947.60$. Conclusion: The average cost of treatment was high in relation to the Brazilian average salary of $\mathrm{R} \$$ 2,110.00 (IBGE-2017). The costs investigated in this study can help public health managers in strategies to rationalize expenditures, optimize capital and maintain patient care.

Key words: Breast Neoplasms/economics; Tamoxifen; Unified Health System.
\end{abstract}

\section{Resumen}

Introducción: La estimación del Instituto Nacional del Cáncer José Alencar Gomes da Silva para Brasil, para cada ańo del bienio 2018-2019, fue de 59,700 casos nuevos de cáncer de mama, con un riesgo estimado de 56.33 casos por 100,000 mujeres. En 2014, los gastos públicos con atención oncológica fueron de aproximadamente R \$ 2,5 mil millones. Objetivo: Evaluar el impacto de los costos directos médicos en pacientes con carcinoma mamario tratados con tamoxifeno por el Sistema Único de Salud. Método: Estudio exploratorio de costo de la enfermedad, cuantitativo, retrospectivo, con carácter de prevalencia y de enfoque bottom-up. La recolección de los datos fue realizada en el Ambulatorio de Oncología del Hospital São Vicente en Curitiba, Paraná. Resultados: El promedio del costo del tratamiento de los pacientes fue $\mathrm{R} \$ 14.497,70$ en tratamiento neoadyuvante o adyuvante y de $\mathrm{R} \$ 9.108,60$ en tratamiento paliativo. En relación a estas variables el costo del tamoxifeno fue el que más impactó en relación al costo total del tratamiento, representando más del $80 \%$ de este valor. El promedio del costo anual gastado con tamoxifeno por paciente fue de R \$ 1.947,60. Conclusión: El costo promedio del tratamiento demostró ser alto en relación al promedio salarial de los brasileńos de R \$ 2.110.00 (IBGE-2017). Los costos levantados en este estudio pueden auxiliar a los gestores de salud pública en estrategias para racionalización de los gastos, optimización del capital y mantenimiento de la atención a la población.

Palabras clave: Neoplasias de la Mama/economía; Tamoxifeno; Sistema Único de Salud.

1 Setor de Ciências da Saúde, Departamento de Farmácia, Universidade Federal do Paraná (UFPR). Curitiba (PR), Brasil. Orcid iD: https://orcid.org/0000-00029451-6758

2 Setor de Ciências da Saúde, Departamento de Farmácia, UFPR. Curitiba (PR), Brasil. Orcid iD: https://orcid.org/0000-0002-4829-1703

${ }^{3}$ Setor de Ciências da Saúde, Departamento de Farmácia, UFPR. Curitiba (PR), Brasil. Orcid iD: https://orcid.org/0000-0001-7810-6831

Endereço para correspondência: Mariangela Adriane Seroiska. Rua Martim Afonso, 371, 403B - São Francisco. Curitiba (PR), Brasil. CEP 80410-060.

E-mail: mari.seroiska@yahoo.com.br 


\section{INTRODUÇÃO}

O carcinoma mamário, ou câncer de mama, é considerado o segundo de maior incidência em todo mundo e o de maior mortalidade entre a populaçáo feminina. A estimativa do Instituto Nacional de Câncer José Alencar Gomes da Silva (INCA) para o Brasil, para cada ano do biênio 2018-2019, foi de 59.700 novos casos de câncer de mama, com um risco estimado de 56,33 casos a cada 100 mil mulheres ${ }^{1}$.

Quando a doença é diagnosticada no início, o tratamento tem maior potencial curativo. Segundo o INCA, na América do Sul, particularmente no Brasil, a sobrevida em cinco anos aumentou de $78 \%$ para $87 \%$ como resultado das políticas públicas adotadas ${ }^{2,3}$.

Segundo os dados do Ministério da Saúde, em 2014, os gastos públicos com atenção oncológica fecharam em aproximadamente $\mathrm{R}$ \$ 2,5 bilhóes. Esses valores correspondem aos gastos com cirurgias, quimioterapia e radioterapia. Entre os anos de 2000 e 2014, esses gastos sofreram um aumento de $357 \%{ }^{4}$.

Segundo o Programa de Controle de Câncer de Mama, o tratamento para o carcinoma mamário varia de acordo com o estadiamento da doença em tratamento local (cirurgia e radioterapia) e sistêmico (quimioterapia, hormonioterapia e terapia biológica). Além disso, as características biológicas e as condiçóes do paciente (idade, status menopausal, comorbidades etc.) também influenciam na escolha da abordagem terapêutica 5 .

No tratamento hormonal, o tamoxifeno é amplamente prescrito para todos os estágios da doença. Segundo as Diretrizes Diagnósticas e Terapêuticas do Carcinoma de Mama do Ministério da Saúde, a utilização do tamoxifeno é indicada nas terapias adjuvante, neoadjuvante e paliativa, nas quais os tumores apresentam receptores hormonais positivos e para os pacientes em pré e pós-menopausa ${ }^{4,6}$.

O tamoxifeno é um modulador seletivo do receptor de estrogênio que inibe o crescimento das células do câncer de mama por antagonismo competitivo de estrogênio. Está associado à maior sobrevida livre e global da doença quando utilizado no tratamento adjuvante, além da redução do câncer de mama contralateral ${ }^{4,6}$.

Os estudos sobre custo da doença (cost of illness), também conhecidos como carga ou ônus da doença (burden of disease), estão entre os primeiros estudos econômicos ligados à saúde identificados na literatura. $\mathrm{O}$ método é uma abordagem empírica para estimar o impacto social das doenças e lesóes, que combina os custos diretos médicos (remuneração profissional, medicamentos, exames complementares, diárias hospitalares), diretos não médicos, (transporte, adaptaçôes domiciliares, cuidados com alimentação e vestimentas), indiretos (perda de produtividade) e os intangíveis (mudanças na qualidade de vida, dor, sofrimento) ${ }^{7-9}$.
A avaliação dos custos de uma doença pode ser realizada sob diferentes perspectivas; ou seja, sob o ponto de vista do paciente, do empregador, da seguradora ou plano de saúde, do governo ou da sociedade. O presente estudo realizou as avaliaçóes dos custos do tratamento do carcinoma mamário com tamoxifeno sob a perspectiva do Sistema Único de Saúde (SUS) ${ }^{10,11}$.

Estudos do tipo custo da doença tornam-se importantes para a saúde pública, pois conseguem quantificar os recursos gastos pelo sistema. O objetivo deste estudo foi avaliar o impacto dos custos diretos médicos em pacientes com carcinoma mamário tratados com tamoxifeno pelo SUS e contribuir como ferramenta de suporte para tomadas de decisóes garantindo tanto o direito individual ao tratamento realmente benéfico quanto o direito de acesso universal ao SUS.

\section{MÉTODO}

Este é um estudo exploratório de custo da doença, quantitativo, retrospectivo, com caráter de prevalência e de abordagem bottom-up.

A coleta de dados foi realizada no Ambulatório de Oncologia do Hospital São Vicente (HSV-FUNEF) em Curitiba, Paraná. A listagem inicial dos pacientes foi obtida por intermédio do relatório de consumo de tamoxifeno por paciente, extraído do sistema utilizado na unidade hospitalar $\left(\mathrm{MV}^{\circledR}\right)$ pelo período de $1^{\circ}$ de janeiro de 2010 a 31 de janeiro de 2016.

Do total de pacientes obtidos na listagem, foram adotados como critério de inclusão pacientes com câncer de mama em tratamento de hormonioterapia com tamoxifeno, com idade superior a 18 anos, atendidos pelo SUS. Foram usados como critérios de exclusão pacientes gestantes, portadores de HIV (buman immunodeficiency virus), em uso de tamoxifeno concomitante à quimioterapia ou radioterapia, com período de tratamento inferior a seis meses, sem dados ou com dados de prontuário incompletos e os pacientes que não assinaram o Termo de Consentimento Livre e Esclarecido (TCLE) por motivo de óbito ou não adesão ao estudo.

Os dados dos participantes da pesquisa foram coletados dos prontuários e registrados em uma ficha clínica desenhada especificamente para o estudo. $\mathrm{Na}$ sequência, os dados retirados das fichas clínicas foram compilados em planilhas em Microsoft Office Excel $^{\circledR}$ e Microsoft Office Word ${ }^{\circledR}$.

Foram coletados dados referentes às características do paciente (informaçóes demográficas, data de diagnóstico da doença, estadiamento, data de início e término do tratamento, comorbidades e outros tratamentos utilizados), da doença (tempo do diagnóstico ao tratamento, escolha do tratamento, regime e posologia, frequência, duração, efeitos colaterais, intervençôes, 
suspensão, alteraçóes, consultas, exames de diagnóstico e internaçôes) e dos recursos utilizados (custo de consultas, exames, procedimentos cirúrgicos, medicamento).

Por se tratar de um estudo sob perspectiva do SUS, foram incluídos apenas os custos diretos médicos, conforme recomendação das Diretrizes Metodológicas para Estudos de Avaliação Econômica de Tecnologias em Saúde ${ }^{11}$. Estes foram a somatória dos custos de consultas médicas e não médicas (multidisciplinares), exames laboratoriais, exames de diagnóstico, medicamentos, procedimentos cirúrgicos, atendimentos em pronto atendimento e unidades de terapia intensiva e internaçóes.

Para o cálculo do valor gasto com consultas, foi utilizada a tabela do Sistema de Gerenciamento da Tabela de Procedimentos, Medicamentos e órteses, próteses e materiais especiais do SUS (SIGTAP-DATASUS), com valores referentes ao mês de outubro de 2017. O valor de cada consulta especificado por essa tabela foi multiplicado pelo número de consultas de cada paciente no período de corte adotado pelo estudo.

$\mathrm{O}$ valor dos exames de diagnóstico e laboratoriais também foram retirados da tabela do SIGTAP e o valor de cada exame, multiplicado pelo número total de exames realizados pelos pacientes dentro do período avaliado.

Para o cálculo dos valores de internação e procedimentos cirúrgicos, foi utilizada a Autorização de Internação Hospitalar emitida para cada paciente no decorrer do período avaliado.

Para análise do custo de medicamentos e demais insumos farmacêuticos, foram utilizados a tabela Brasíndice e o valor de custo médio obtido no sistema hospitalar utilizado pela instituição $\left(\mathrm{MV}^{\circledR}\right)$.

Os custos avaliados foram separados em grupos: medicamentos, consultas, exames, cirurgias e internaçóes (Microsoft Office Excel ${ }^{\circledR}$ ); comparação entre pacientes que realizaram algum procedimento cirúrgico durante o tratamento (cirúrgico e não cirúrgico) para avaliação das diferenças de custos entre eles; comparação entre os tipos de tratamento (adjuvante e paliativo) para avaliaçáo das diferenças de custos entre eles.

Algumas variáveis foram selecionadas para se correlacionem com os custos por meio do coeficiente de correlação de postos de Spearman's Rho. Os dados referentes ao perfil dos pacientes e custos do carcinoma mamário foram analisados por estatística descritiva e testes de associação. A escolha dos testes estatísticos foi baseada na distribuição e normalidade das variáveis utilizando o teste Kolmogorov-Smirnov. Em caso de distribuição normal, as variáveis foram apresentadas como média e desvio-padráo e aplicou-se o teste t de Student. Em caso de distribuição não normal, os valores foram expressos como mediana e range, sendo utilizados os testes de Mann-Whitney e Kruskal-Wallis. Quando pertinente, foi empregado o teste de qui-quadrado para comparação.
Todas as análises estatísticas foram realizadas com auxílio do programa estatístico SPSS v.17.0, sendo considerados estatisticamente significativos resultados com valor de $\mathrm{p}<0,05$.

Este estudo foi aprovado pelo Comitê de Ética em Pesquisa em Seres Humanos (CEP) do Setor de Ciências da Saúde da Universidade Federal do Paraná, protocolo de registro CEP/SD - PB: 1912874/ 2017-02. Foi aplicado o TCLE para o total de pacientes da amostra que atenderam aos critérios de inclusão e não se enquadraram nos critérios de exclusão descritos no estudo. Um total de 176 pacientes assinaram o TCLE.

\section{RESULTADOS}

$\mathrm{Na}$ listagem inicial, foram obtidos 815 pacientes submetidos ao tratamento de hormonioterapia com tamoxifeno. A partir dessa listagem, 639 foram excluídos por não atenderem aos critérios de inclusão ou apresentarem algum dos critérios de exclusão citados no estudo. $\mathrm{O}$ resultado da amostragem foi de 176 pacientes.

Os principais critérios de exclusão evidenciados foram a associação de quimioterapia ou radioterapia ao tratamento, alteração de tratamento durante o período de corte do estudo em razão da troca de tratamento por progressão da doença, os que possuíam prontuários incompletos ou não assinaram o TCLE.

Os dados sociodemográficos foram compilados em tabela única (Tabela 1), na qual é possível evidenciar que a maioria dos pacientes do estudo foi de mulheres na faixa etária média de 65 anos.

O tempo médio de tratamento foi de 55 meses (desvio-padrão \pm 14 meses). Em relação aos exames do seguimento terapêutico dos pacientes, à classificação BI-RADS ${ }^{\circledR}$ de diagnóstico, 51,1\% dos pacientes apresentaram diagnóstico de BI-RADS ${ }^{\circledR} 2$, seguido de $25 \%$ apresentando BI-RADS ${ }^{\circledR} 0$. A esteatose hepática demonstrou ser a comorbidade mais relevante associada ao uso do tamoxifeno $(26,1 \%)$, enquanto a hipertensão arterial sistêmica $(38,9 \%)$ e o diabetes mellitus $(13,9 \%)$ demonstraram ser as comorbidades de maior ocorrência não associadas ao uso do tamoxifeno. Entre os pacientes que apresentaram metástase secundária, a metástase pulmonar e a de múltiplos órgãos, percentualmente equivalentes $(1,7 \%)$, foram as mais frequentes.

A média do número de consultas médicas realizadas por paciente durante o período de tratamento foi de 16 consultas para pacientes em tratamento adjuvante e 13 consultas para os pacientes em tratamento paliativo (Tabela 2).

Observa-se uma média de consultas médicas de 3,5 consultas/ano para os pacientes em tratamento adjuvante e 2,8 consultas/ano para os pacientes em tratamento paliativo. Não houve diferença significativa na média de 
Tabela 1. Variáveis sociodemográficas

\begin{tabular}{lc}
\hline \multicolumn{1}{c}{ Variável } & $\mathbf{n}(\%)$ \\
\hline Sexo & $2(1,1)$ \\
Masculino & $174(98,9)$ \\
Feminino & \\
Estado Civil & $65(36,9)$ \\
Casado & $11(6,3)$ \\
Divorciado & $2(1,1)$ \\
Não declarado & $1(0,6)$ \\
Separado & $67(38,1)$ \\
Solteiro & $30(17,0)$ \\
Viúvo & \\
Raça & $171(97,2)$ \\
Branca & $4(2,3)$ \\
Negra & $1(0,6)$ \\
Parda & \\
Grau de escolaridade & $22(12,5)$ \\
Ensino fundamental completo & $6(3,4)$ \\
Ensino fundamental incompleto & $18(10,2)$ \\
Ensino médio completo & $8(4,5)$ \\
Ensino superior completo & $1(0,6)$ \\
Alfabetizado & $1(0,6)$ \\
Mestrado & $120(68,2)$ \\
Não declarado & \\
Ocupação & $49(27,8)$ \\
Trabalha & $125(71,0)$ \\
Não trabalha & $2(1,1)$ \\
Não declarado & \\
Localidade & $131(74,4)$ \\
Capital & $27(15,3)$ \\
Região Metropolitana & $18(10,3)$ \\
Outros & \\
\hline
\end{tabular}

consultas médicas entre os pacientes dos diferentes grupos de tratamento. A especialidade médica com o maior número de consultas ambulatoriais foi a oncologia, com um número médio de 3,4 consultas/ano e um desvio-padráo de $\pm 2,2$. Essa especialidade representou $85,7 \%$ do número total de consultas médicas. Entre as demais especialidades médicas, estão a clínica geral, dermatologia, endocrinologia, cardiologia, entre outras.

Apenas 26 pacientes (14,7\%) realizaram consultas não médicas durante o período do estudo. A média do número de consultas não médicas, para esses pacientes, foi de 0,81 consultas/ano, com um desvio-padráo de $\pm 0,59$. Entre os profissionais multidisciplinares consultados, estão: nutricionista, psicólogo, assistente social, farmacêutico, fisioterapeuta e a equipe de enfermagem. Identificaram-se uma subnotificação dos registros de consultas multidisciplinares nos prontuários e a náo existência de repasse dos valores da maioria das consultas consulta da fisioterapia e enfermagem. A consulta farmacêutica representou $79 \%$ do total de consultas não médicas realizadas, sendo estas relacionadas à orientação sobre o uso do medicamento.

Um total de 50 pacientes $(28,4 \%)$ realizou consultas de pronto atendimento durante o período do estudo. A média do número dessas consultas para esses pacientes foi de 0,53 consultas/ano, com um desvio-padrão de $\pm 0,4$. O motivo das consultas realizadas foi variado, sendo na sua grande maioria de clínica geral de comorbidades não relacionadas ao tratamento.

O valor médio dos gastos com consultas médicas para os pacientes em tratamento paliativo, assim como no tratamento adjuvante, correspondeu a $1,5 \%$ do valor total gasto com o tratamento, e o valor médio gasto com consultas não médicas demonstrou ser inferior a $0,1 \%$ do valor total do tratamento. $\mathrm{O}$ valor pago por consulta médica ambulatorial em atenção especializada foi de $\mathrm{R} \$$ 10,00 . O valor unitário pago pela consulta de profissionais de nível superior (exceto médico) no ambulatório de atenção especializada foi de $R \$ 6,30$. Esses valores foram descritos conforme a tabela SIGTAP (out./2017). O valor médio gasto com consultas do pronto atendimento tanto para os pacientes em tratamento adjuvante quanto em tratamento paliativo foi inferior a $0,5 \%$ em relação ao custo total do tratamento.

A média do valor gasto com consultas médicas e não médicas representou um valor menor que 2,0\% do custo total do tratamento tanto para os pacientes que realizaram algum procedimento cirúrgico como para os que não realizaram. Houve diferença significativa de valores gastos com consultas não médicas entre os pacientes que realizaram procedimento cirúrgico e os que nâo realizaram $(\mathrm{p}<0,05)$.

$\mathrm{O}$ valor médio gasto com exames de diagnóstico pelos pacientes em tratamentos adjuvante e paliativo corresponderam respectivamente a $3,78 \%$ e $4,34 \%$ do

Tabela 2. Comparativo do número total de consulta e exames entre grupos de tratamento

\begin{tabular}{lccccccc}
\hline \multirow{2}{*}{ consULTAS E EXAMES } & \multicolumn{3}{c}{ ADJUVANTE } & \multicolumn{3}{c}{ PALIATIVO } \\
\cline { 2 - 7 } & Total & Média & Mediana & Total & Média & Mediana \\
\hline Consultas médicas & 2.673 & 16,10 & 16 & 125 & 12,5 & 15 \\
Consultas não médicas & 81 & 10 & 0 & 14 & 11 & 0,5 \\
Consultas de pronto atendimento & 110 & 66 & 0 & 7 & 47 & 0 \\
Exames diagnóstico & 1.696 & 322 & 10 & 91 & 318 & 7 \\
Exames laboratório & 1.739 & 39 & 5 & 52 & 24 & 0 \\
\hline
\end{tabular}


valor médio do custo do tratamento. Os pacientes que realizaram procedimento cirúrgico apresentaram uma média de gasto com exames de diagnóstico por ano de $\mathrm{R} \$$ 117,27 exames/ano, com um desvio-padrão de $\pm 70,22$. A média de gasto com exames de laboratório foi de $\mathrm{R} \$$ 10,43 exames/ano com um desvio-padrão de $\pm 9,13$. A média do custo de exames de diagnóstico e de laboratório corresponderam um valor $\leq 1,00 \%$ da média do custo do tratamento. Não houve correlação significativa dos valores de custos de exames laboratoriais e de diagnóstico entre os pacientes que realizaram procedimento cirúrgico e os que não realizaram.

Os exames de diagnóstico mais solicitados foram a mamografia, ultrassonografia de mama, radiografia de tórax e ultrassonografia de abdômen total. Avaliando o número de mamografias realizadas por paciente durante o tratamento, tem-se 0,59 exames/paciente/ano, com desvio-padrão de $\pm 0,4$. Assim, a média do custo com mamografia foi de $\mathrm{R} \$ 26,77$ exames/ano, com um desvio-padrão de $\pm 18,05$. O valor unitário pago pelo exame foi de $\mathrm{R} \$ 45,00$, de acordo com a tabela SIGTAP (out./2017).

O número médio de ultrassonografias de mama solicitadas foi de 0,51 exames/paciente/ano, com desvio-padrão de $\pm 0,36$. A média do valor gasto em ultrassonografia de mama durante o tratamento foi de $\mathrm{R} \$ 12,50$ exames/ano, com um desvio-padrão de $\pm 8,62$, o que corresponde a um custo médio anual de $\mathrm{R} \$ 10,28$. O valor unitário pago pelo exame é de $\mathrm{R} \$ 24,20$, conforme a tabela SIGTAP (out./2017).

O número médio de exames de radiografia de tórax solicitados foi de 0,73 exames/paciente/ano, com desvio-padrão de $\pm 0,68$. A média do valor gasto em radiografia de tórax foi de $\mathrm{R} \$ 5,00$ paciente/ano, com um desvio-padrão de $\pm 4,65$, o que corresponde a um gasto médio anual de $\mathrm{R} \$ 3,95$. O valor unitário pago pela radiografia de tórax, conforme a tabela SIGTAP (out./2017), foi de R \$ 6,88.

$\mathrm{O}$ número médio de ultrassonografias de abdômen total foi de 0,58 exames/paciente, com desvio-padrão de $\pm 0,48$. A média do valor gasto durante o tratamento foi de $\mathrm{R} \$ 82,37$ ultrassonografias/ano, isto corresponde a um gasto médio anual de $\mathrm{R} \$ 21,40$ paciente/ano, com um desvio-padrão de $\pm 18,09$. O valor unitário pago pelo exame segundo a tabela SIGTAP (out./2017) foi de R \$ 37,95 .

O número médio de exames laboratoriais solicitados durante o período do tratamento foi de 10,27 exames, com um desvio-padrão de $\pm 13,80$. O número médio de exames laboratoriais foi de 2,24 exames/ano. O exame laboratorial mais frequentemente solicitado foi o hemograma, com uma média de solicitação de 0,05 exames/ano, com um desvio-padrão de $\pm 0,05$. $O$ valor médio gasto por ano foi de $\mathrm{R} \$ 2,41$, com um desvio-padrão de $\pm 2,5$. O valor unitário pago pelo hemograma, conforme tabela do SIGTAP (out/2017), foi de R \$ 4,11.

O valor médio do custo com procedimentos cirúrgicos para os pacientes que realizaram algum procedimento cirúrgico durante o período do estudo, independentemente do tipo de tratamento, foi de $\mathrm{R} \$$ 732,30 procedimentos/ano, com um desvio-padrão de $\pm 580,17$. Foi possível evidenciar que o valor médio do custo com cirurgias durante o período do estudo para os pacientes que realizaram tais procedimentos correspondeu a $25,43 \%$ do valor médio do custo total do tratamento desses pacientes. E que houve diferença estatística significativa nas variáveis custo de cirurgias e custo total do tratamento nos pacientes que realizaram procedimento cirúrgico. Essas variáveis são diretamente correlacionadas (Tabela 3).

A média do custo com tamoxifeno durante o período do tratamento para os pacientes deste estudo foi de $\mathrm{R} \$$ $8.924,66$, com um desvio-padrão de $\pm 2.265,83$. A média do custo anual com tamoxifeno foi de $\mathrm{R} \$ 1.947,60$, independentemente do tipo de tratamento ou da realização de procedimento cirúrgico.

Comparando os pacientes que realizaram ou náo algum procedimento cirúrgico, é possível evidenciar que os pacientes cirúrgicos apresentaram a média do gasto com tamoxifeno durante o tratamento $14 \%$ maior

Tabela 3. Comparativo de custos de pacientes submetidos a procedimentos cirúrgicos versus pacientes não submetidos a procedimentos cirúrgicos

\begin{tabular}{|c|c|c|c|c|c|}
\hline \multirow[b]{2}{*}{ Custo (RS) } & \multicolumn{2}{|c|}{ COM CIRUGIA } & \multicolumn{2}{|c|}{ SEM CIRURGIA } & \multirow[b]{2}{*}{ p-valor } \\
\hline & $\begin{array}{l}\text { Média* } \\
\text { ( } \pm \text { DP) }\end{array}$ & $\begin{array}{l}\text { Mediana } \\
\text { (25-75) }\end{array}$ & $\begin{array}{l}\text { Média* } \\
\text { (土DP) }\end{array}$ & $\begin{array}{l}\text { Mediana } \\
(25-75)\end{array}$ & \\
\hline Exames de diagnóstico & $\begin{array}{c}146,00 \\
(75)\end{array}$ & $\begin{array}{c}14.000 \\
(90-200)\end{array}$ & $\begin{array}{c}331,60 \\
(283)\end{array}$ & $\begin{array}{c}255,40 \\
(114-462)\end{array}$ & 0,17 \\
\hline Exames de laboratório & $2,90(7)$ & $\begin{array}{c}0 \\
(0-0)\end{array}$ & $\begin{array}{c}20,40 \\
(32)\end{array}$ & $\begin{array}{c}3,50 \\
(0-31)\end{array}$ & 0,59 \\
\hline Custo do tratamento & $\begin{array}{c}14.497,70 \\
(3.196)\end{array}$ & $\begin{array}{c}13.417,80 \\
(12.198-15.433)\end{array}$ & $\begin{array}{c}9.108,6 \\
(2.452,96)\end{array}$ & $\begin{array}{c}9.9378,80 \\
(10.328)\end{array}$ & 0,00 \\
\hline
\end{tabular}

Legenda: $\mathrm{DP}=$ desvio-padrăo.

Notas: Correlaçấo significativa em p-valor $<0,05$ (Wilcox-Mann-Whitney ${ }^{\circledR}$ ); * do tratamento completo. 
do que os pacientes que não realizaram procedimento cirúrgico. Esse fato pode ser correlacionado ao custo de o tratamento total ser superior nesse grupo em virtude dos custos agregados aos procedimentos cirúrgicos. Para os pacientes que realizaram algum procedimento cirúrgico durante o tratamento, a média do custo do tratamento com tamoxifeno representou 68,71\% da média do custo total do tratamento e $94,06 \%$ para os pacientes que não realizaram nenhum procedimento cirúrgico (Tabela 4). Evidenciou-se que os pacientes que não realizaram procedimento cirúrgico eram na maioria idosos e/ou apresentavam quadro metastático da doença.
Em relação a outras variáveis de custo analisadas neste estudo, o custo do tamoxifeno foi o que mais impactou em relação ao custo total do tratamento. A média do custo do tratamento com tamoxifeno representou mais de $80 \%$ do valor médio do total do tratamento dos pacientes, exceto no grupo de que realizou algum procedimento cirúrgico durante o tratamento.

Segundo as análises estatísticas realizadas entre as variáveis, as que apresentaram uma forte correlação em relação ao custo do tratamento, objeto deste estudo, foram o tempo do tratamento, o custo das cirurgias e o custo do tamoxifeno, conforme demonstra a Tabela 5.

Tabela 4. Custo do tratamento com tamoxifeno em relação aos pacientes que realizaram algum procedimento cirúrgico

\begin{tabular}{|c|c|c|c|c|c|}
\hline \multirow[b]{2}{*}{ Custo (RS) } & \multicolumn{2}{|c|}{ COM CIRUGIA } & \multicolumn{2}{|c|}{ SEM CIRURGIA } & \multirow[b]{2}{*}{ p-valor } \\
\hline & $\begin{array}{l}\text { Média* } \\
\text { (土DP) }\end{array}$ & $\begin{array}{c}\text { Mediana } \\
(25-75)\end{array}$ & $\begin{array}{l}\text { Média* } \\
\text { (土DP) }\end{array}$ & $\begin{array}{c}\text { Mediana } \\
\text { (25-75) }\end{array}$ & \\
\hline \multirow{2}{*}{ Custos do tamoxifeno } & $9.961,60$ & $9.738,00$ & $8.568,40$ & $9.575,70$ & \multirow{2}{*}{0,00} \\
\hline & $(1.452)$ & $(9.738-9.738)$ & $(2.387)$ & (7.953-9.738) & \\
\hline \multirow{2}{*}{ Custo do tratamento } & $14.497,70$ & $13.417,80$ & $9.108,6$ & $9.9378,80$ & \multirow{2}{*}{0,00} \\
\hline & (3.196) & (12.198-15.433) & $(2.452,96)$ & (10.328) & \\
\hline
\end{tabular}

Legenda: $\mathrm{DP}=$ desvio-padrăo.

Notas: Correlaçáo significativa em p-valor <0,05 (Wilcox-Mann-Whitney ${ }^{\circledR}$ ); * do tratamento completo.

Tabela 5. Correlações estatísticas entre variáveis (Spearman's Rho)

\begin{tabular}{|c|c|c|c|c|c|c|c|c|c|c|}
\hline & $\begin{array}{l}\text { Tempo de } \\
\text { tratamento }\end{array}$ & BI-RADS $®$ & $\begin{array}{l}\text { Custo das } \\
\text { consultas } \\
\text { médicas }\end{array}$ & $\begin{array}{c}\text { Custo das } \\
\text { consultas } \\
\text { não médicas }\end{array}$ & $\begin{array}{l}\text { Custo dos } \\
\text { exames de } \\
\text { diagnóstico }\end{array}$ & $\begin{array}{l}\text { Custo dos } \\
\text { exames de } \\
\text { laboratório }\end{array}$ & $\begin{array}{l}\text { Custo de } \\
\text { cirurgias }\end{array}$ & $\begin{array}{l}\text { Custo das } \\
\text { consultas } \\
\text { de pronto } \\
\text { atendimento }\end{array}$ & $\begin{array}{c}\text { Custo } \\
\text { total do } \\
\text { tamoxifeno }\end{array}$ & $\begin{array}{c}\text { Custo } \\
\text { total do } \\
\text { tratamento }\end{array}$ \\
\hline \multicolumn{11}{|l|}{$\begin{array}{l}\text { Tempo de } \\
\text { tratamento } \\
\text { (meses) }\end{array}$} \\
\hline BI-RADS $®$ & 0,066 & & & & & & & & & \\
\hline $\begin{array}{l}\text { Custo das } \\
\text { consultas } \\
\text { médicas }\end{array}$ & $-0,363^{* *}$ & 0,038 & & & & & & & & \\
\hline $\begin{array}{l}\text { Custo das } \\
\text { consultas não } \\
\text { médicas }\end{array}$ & 0,069 & 0,007 & 0,025 & & & & & & & \\
\hline $\begin{array}{l}\text { Exames de } \\
\text { diagnóstico }\end{array}$ & $0,266^{* *}$ & $0,193^{*}$ & $-0,040$ & 0,041 & & & & & & \\
\hline $\begin{array}{l}\text { Exames de } \\
\text { laboratório }\end{array}$ & $0,365^{* *}$ & 0,147 & $-0,081$ & 0,017 & $0,422^{* *}$ & & & & & \\
\hline $\begin{array}{l}\text { Custo de } \\
\text { cirurgias }\end{array}$ & $0,405^{* *}$ & 0,126 & $-0,101$ & 0,027 & $0,373^{* *}$ & $0,367^{* *}$ & & & & \\
\hline $\begin{array}{l}\text { Custo das } \\
\text { consultas } \\
\text { de pronto } \\
\text { atendimento }\end{array}$ & 0,079 & 0,005 & $-0,142$ & 0,059 & $0,210^{* *}$ & $0,246^{* *}$ & $0,197^{* *}$ & & & \\
\hline $\begin{array}{l}\text { Custo total do } \\
\text { tamoxifeno }\end{array}$ & $1,000^{* *}$ & 0,066 & $-0,363^{* *}$ & 0,069 & $0,266^{* *}$ & $0,365^{* *}$ & $0,405^{* *}$ & 0,079 & & \\
\hline $\begin{array}{l}\text { Custo total do } \\
\text { tratamento }\end{array}$ & $0,815^{* *}$ & $0,150^{*}$ & $-0,198^{* *}$ & 0,050 & $0,557^{* *}$ & $0,478^{* *}$ & $0,743^{* *}$ & $0,204^{* *}$ & $0,815^{* *}$ & \\
\hline
\end{tabular}

Notas: Correlação de Spearman (Rho); ${ }^{*}$ p-valor<0,05; ${ }^{* *}$ p-valor<0,001. Na correlação, o sig indica o p-valor e o coeficiente de correlação indica a força da correlação entre as variáveis. O sinal desse coeficiente indica se a correlação é positiva (ambas as variáveis aumentam no mesmo sentido) ou negativa (quando uma das variáveis aumenta, a outra diminui). P-valor $<0,05$ é significativo, e a interpretação do coeficiente de correlação é a seguinte: 0,00 a 0,19 - correlação bem fraca; 0,20 a 0,39 - correlação fraca; 0,40 a 0,69 - correlação moderada; 0,70 a 0,89 - correlação forte; 0,90 a 1,00 - correlação muito forte. 
Comparando os custos conforme o tipo de tratamento, observa-se que o tratamento tipo 1 teve maior custo que o 2 em relação ao custo do tamoxifeno e do tratamento total.

\section{DISCUSSÃO}

A variável tempo do tratamento apresentou uma forte correlação em relaçáo ao custo total do tratamento (Rho $0,815)$. Essas duas variáveis possuem uma correlação diretamente proporcional, tendo em vista o fato de que quanto maior for o tempo do tratamento, maior será o seu custo. A média do tempo de tratamento do estudo foi de 55 meses. Os valores encontram-se próximos aos descritos nos estudos de Guedes et al. ${ }^{12}$, Brito et al. ${ }^{13} \mathrm{e}$ Oliveira et al..$^{14}$.

Segundo os Protocolos Clínicos e Diretrizes Terapêuticas em Oncologia, o tempo de tratamento com tamoxifeno recomendado é de cinco anos (60 meses), mas atuais evidências sugerem um protocolo de dez anos (120 meses) para alguns casos. Dos pacientes que iniciaram o tratamento durante o período deste estudo, $49,4 \%$ completaram cinco anos de tratamento conforme preconizado $^{14}$. A média do tempo de tratamento deste estudo foi de 4,6 anos.

As vantagens da hormonioterapia, incluindo o aumento da sobrevida, são clinicamente evidentes e o acesso gratuito à medicação no SUS contribui para esse bom prognóstico. Estudos garantem que a redução das taxas de mortalidade e a recorrência da doença são eficazes no cumprimento integral do tempo de tratamento. Entretanto, os resultados, muitas vezes, não são alcançados por causa do náo cumprimento do tempo total do tratamento. Uma das principais causas citadas é o tempo prolongado da terapia associado à ausência do tumor e/ ou ao sintomas ${ }^{12,14}$.

Em relação ao tempo de tratamento, a média do custo total do tratamento para os pacientes com tempo de tratamento $\geq 55$ meses foi de $\mathrm{R} \$ 2.316,13$ tratamento/ ano, com um desvio-padrão de $\pm 506,60$. Esse valor é $6,55 \%$ maior que a média do custo do tratamento para os pacientes com tempo de tratamento $<55$ meses, que foi de $\mathrm{R} \$ 2.143,66$ tratamento/ano com um desvio-padrão de $\pm 126,60$.

A idade é um dos principais fatores de risco para o câncer de mama, com risco de morte aumentado nos tumores diagnosticados após os 50 anos de idade ${ }^{2,4,15}$. A faixa etária média obtida neste estudo diverge dos estudos de Leite et al. ${ }^{6}$ e de Aguiar et al. ${ }^{16}$, nos quais a idade média esteve na faixa entre 40 e 60 anos. Entretanto, encontra-se em equivalência com o estudo de Haddad et al. ${ }^{17}$, em que a faixa etária da maioria da população foi de 50 a 69 anos. Neste estudo, a idade mínima foi de 25 anos e a máxima de 95 anos.
A categoria BI-RADS ${ }^{\circledR} 2$ é indicativa de achados benignos cuja conduta é o controle de rotina nos casos de nódulos sólidos e de características benignas estáveis por mais de dois anos, linfonodos intramamários, implantes, alteraçôes arquiteturais após procedimentos cirúrgicos; características estas evidenciadas nos pacientes em tratamentos adjuvante e paliativo deste estudo ${ }^{18}$. Os resultados obtidos neste estudo nas categorias BIRADS $^{\circledR}$ apresentaram-se próximos aos dados obtidos no estudo de Rocha ${ }^{19}$.

Em relaçáo às comorbidades associadas ao uso de tamoxifeno, a esteatose hepática demonstrou ser a mais relevante. Esse dado encontra-se muito próximo ao citado no estudo de El-Beshbishy, que foi de 30\%. A ocorrência de alteraçóes hepáticas é descrita em literatura em mais de $30 \%$ dos pacientes que usam tamoxifeno. Entre essas alterações, estão incluídas a hepatite tóxica, a esteatose hepática não alcoólica e a necrose. Apesar de sua ação antiestrogênica, o tamoxifeno possui ações agonistas ao estrogênio nos receptores do fígado e no metabolismo lipídico, o que ocasionaria essas alteraçôes hepáticas descritas ${ }^{20,21}$.

As variáveis tempo de tratamento e custo das consultas médicas apresentaram uma correlação fraca e inversamente proporcional (Rho 0,363). Nesse caso, não é possível afirmar que o uso do medicamento por um tempo maior de tratamento diminuiria o número de consultas médicas, visto que o protocolo e a necessidade de renovação das Autorizaçôes de Procedimento de Alta Complexidade junto à Secretaria Municipal de Saúde, para a liberação do tamoxifeno, preconizam a realizaçáo de uma consulta com intervalo mínimo de quatro meses. Não existe um consenso em literatura sobre a frequência do número de consultas necessárias para acompanhamento dos pacientes em hormonioterapia. O Consenso e os Protocolos e Diretrizes Terapêuticas em Oncologia preconizam uma média de acompanhamento de três a seis meses para a maioria dos casos. Fatores limitantes deste estudo, como o caso dos pacientes que realizavam acompanhamento externo com outros profissionais do sistema de saúde complementar ou particular, podem ter contribuído para esse valor de correlação $0^{4,8}$.

Náo foram encontrados relatos detalhados em literatura de valores de custo de consultas médicas e não médicas em pacientes com carcinoma mamário em tratamento exclusivamente hormonioterápico, o que dificultou a realização de análises comparativas de custos.

Foi possível evidenciar a forte correlaçáo entre o custo de procedimentos cirúrgicos e o custo total do tratamento (Rho 0,743). A correlação entre essas duas variáveis apresenta-se diretamente proporcional, sendo que os pacientes que realizaram algum procedimento cirúrgico apresentaram a média do custo total do tratamento superior aos pacientes que não realizaram nenhum 
procedimento cirúrgico, conforme dados analisados anteriormente.

Os dados deste estudo diferem dos dados obtidos por Kaliks et al. ${ }^{22}$, no qual o custo médio por paciente gasto com mastectomia + setorectomia foi de R \$ 12.125,00. No entanto, o estudo abrange pacientes em vários estágios da doença e não possui os mesmos critérios adotados no estudo conduzido por nosso grupo, o que dificulta uma análise comparativa. Não se encontraram estudos que relacionassem o custo de procedimentos cirúrgicos em pacientes exclusivamente hormonioterápicos ${ }^{22}$.

$\mathrm{O}$ número de mamografias realizadas por paciente durante o período do estudo foi de 0,59 exames/paciente/ ano, com desvio-padrão de $\pm 0,4$. Evidenciou-se que a maioria dos pacientes apresentou um seguimento terapêutico com solicitação anual de mamografia e para os que apresentaram quadro metastático da doença com solicitação semestral ${ }^{6}$.

No estudo de Kaliks et al. ${ }^{22}$, o custo médio por pacientes com radiografia de tórax e ultrassonografia de abdômen foi de R \$ 651,00. No estudo de custo-efetividade de Peregrino et al. ${ }^{23}$, o custo das mamografias variou entre $\mathrm{R} \$ 29,69$ (histórico natural da doença partir dos 40 anos) até $R \$ 146,60$ (mamografia rastreamento anual a partir dos 40 anos e bianual dos 50-69 anos).

A correlaçáo entre as variáveis custo total do tratamento e o custo do tamoxifeno demonstrou ser forte (Rho 0,815 ) e diretamente proporcional. Quanto maior o tempo do tratamento com tamoxifeno, maior o seu custo e o impacto desse no custo total do tratamento. $\mathrm{O}$ tratamento com o tamoxifeno recomendado, conforme o documento do Consenso para Controle do Câncer de Mama e os Protocolos Clínicos e Diretrizes Terapêuticas em Oncologia, é de cinco anos (60 meses) com possibilidade de extensão da terapia para dez anos (120 meses), segundo estudos recentes. Conforme análise anterior, a média do tempo de tratamento deste estudo foi de 55,0 meses ou 4,6 anos ${ }^{5,8}$

Já a correlação entre o tempo do tratamento e o custo do tamoxifeno demonstrou ser muito forte (Rho 1,00). Os pacientes com tempo de tratamento $\geq 60$ meses foram os que apresentaram as maiores médias de valor em relação ao custo do tamoxifeno e, consequentemente, do custo total do tratamento. Conforme analisado anteriormente, a média do custo do tamoxifeno representou mais de $80 \%$ do custo total do tratamento para os pacientes deste estudo. Estudos demonstram que quanto maior o tempo de tratamento, maior a taxa de sobrevida a longo prazo e maior a qualidade de vida dos pacientes ${ }^{12,13}$.

No trabalho de Kaliks et al. ${ }^{22}$, o custo anual do tratamento com tamoxifeno foi de $\mathrm{R} \$ 1.095,00$ por paciente. Já no estudo de Sasse et al. ${ }^{3}$, o custo semestral do tamoxifeno para pacientes do SUS (incluindo a droga, exames e eventos adversos) seria de $\mathrm{R} \$ 563,67$. Os valores usados pelos autores referem-se às tabelas igualmente utilizadas neste estudo, porém do ano de $2005^{3,22}$.

Não foi possível realizar modelagem de regressáo linear para os custos, pois eles não apresentaram distribuição normal, sendo este um dos requisitos para realização do teste.

A principal limitação deste estudo esteve na náo descrição dos dados em prontuário. Vários pacientes foram excluídos da amostra por falta de dados que comprovassem o diagnóstico, como, por exemplo, resultado do laudo de exames e ausência de dados, como o estadiamento, impossibilitaram a análise comparativa com outras referências bibliográficas.

Outro fator limitante e de exclusão de vários pacientes da amostra foram os prontuários de pacientes que realizavam a consulta médica no ambulatório para retirar o tamoxifeno pelo SUS na Farmácia do Ambulatório de Oncologia, mas realizavam acompanhamento externo com outros profissionais por meio do sistema de saúde complementar ou particular.

Os resultados do presente estudo refletem os dados de uma instituição filantrópica brasileira, mas deve-se ter cautela em extrapolar esses dados para o âmbito nacional.

Foram utilizados os custos do Brasíndice ${ }^{\circledR}$ para o tamoxifeno em razão da variação dos preços nas cotaçóes realizadas pela instituiçáo, resultante de negociaçóes e/ou contratos. Os resultados desse custo podem variar entre instituições.

O fato de não terem sido encontradas publicaçôes com as mesmas variáveis adotadas por este estudo dificultou a realização de análises comparativas de alguns dos resultados obtidos, conforme mencionado no decorrer do texto.

\section{CONCLUSÃO}

O presente estudo de custo da doença traçou os perfis clínico e farmacoterapêutico e estratificou os custos médico diretos relacionados ao tratamento durante o período do estudo, bem como a influência de fatores ligados ao perfil do paciente que poderiam influenciar nos custos médicos diretos como idade, sexo, comorbidades não associadas ao uso de tamoxifeno, entre outros.

As avaliações econômicas, em economia da saúde, visam a auxiliar os gestores da saúde na tomada de decisão quanto à incorporação de tecnologias em saúde, como medicamentos, equipamentos e procedimentos técnicos, sistemas organizacionais, sistemas educacionais, sistemas de informação e de suporte e os programas e protocolos assistenciais, por meio dos quais a atenção e os cuidados sanitários são prestados à população ${ }^{24}$.

Sendo assim, os resultados obtidos no presente estudo, analisados sob a perspectiva do SUS, podem ser utilizados por tais gestores como ferramenta de suporte para tomadas 
de decisóes em relação à incorporação de tecnologias em saúde, garantindo tanto o direito individual ao tratamento realmente benéfico quanto o direito de acesso universal ao SUS.

\section{CONTRIBUIÇÕES}

Mariangela Adriane Seroiska contribuiu substancialmente na concepção, no planejamento, na obtenção, na análise e interpretação dos dados, na redação do estudo e aprovação final da versão publicada. Luana Lenzi contribuiu na análise, interpretação dos dados, revisão crítica e aprovação final da versão publicada. Astrid Wiens contribui na concepção, no planejamento, na análise e interpretação dos dados, na revisão crítica e aprovação final da versão publicada.

\section{AGRADECIMENTOS}

Ao Programa de Pós-Graduação em Ciências Farmacêuticas da Universidade Federal do Paraná e ao Programa de Pós-Graduação em Assistência Farmacêutica (em rede) da Universidade Federal do Rio Grande do Sul (PPGASFAR-UFRGS). A todos os professores e aos colegas das diversas disciplinas presenciais e em rede. Ao Hospital São Vicente, na pessoa do seu Diretor-Técnico Dr. Angelo Tesser pelas informaçóes cedidas e pela oportunidade de aprendizado. A todos os colaboradores e aos colegas de trabalho do Hospital São Vicente, por todo apoio e auxílio na emissão de relatórios e demais informaçôes necessárias.

\section{DECLARAÇÃO DE CONFLITO DE INTERESSES}

Nada a declarar.

\section{FONTES DE FINANCIAMENTO}

Não há.

\section{REFERÊNCIAS}

1. Instituto Nacional de Câncer José Alencar Gomes da Silva. Estimativa 2018: incidência de câncer no Brasil. Rio de Janeiro: INCA; 2017.

2. Instituto Nacional de Câncer José Alencar Gomes da Silva. Estimativa 2016: incidência de câncer no Brasil. Rio de Janeiro: INCA; 2015.

3. Sasse AD, Sasse EC. Estudo de custo-efetividade do anastrozol adjuvante no câncer de mama em mulheres pós-menopausa. Rev Assoc Med Bras. 2009;55(5):535-40. doi: http://dx.doi.org/10.1590/ S0104-42302009000500015.
4. Ministério da Saúde (BR). Protocolos clínicos e diretrizes terapêuticas em oncologia. Brasília, DF: Ministério da Saúde; 2014.

5. Ministério da Saúde (BR). Portaria no 1.008, de 30 de setembro de 2015. Aprova as Diretrizes Diagnósticas e Terapêuticas do Carcinoma de Mama. Diário Oficial da União, Brasília, DF; 2015 out. 1. Seção I, p. 101.

6. Leite FMC, Bubach S, Amorim MHC, et al. Mulheres com diagnóstico de câncer de mama em tratamento com tamoxifeno: perfil sociodemográfico e clínico. Rev Bras Cancerol. 2011;57(1):15-21.

7. Oliveira ML, Santos LMP, Silva EN. Bases metodológicas para estudos de custos da doença no Brasil. Rev. Nutr.2014;27(5):585-595. doi: http://dx.doi. org/10.1590/1415-52732014000500007.

8. Ministério da Saúde (BR). Avaliação econômica em saúde: desafios para gestão no Sistema Único de Saúde. Brasília, DF: Ministério da Saúde; 2008. (Série A. Normas e Manuais Técnicos).

9. GBD 2016 Brazil Collaborators. Burden of disease in Brazil, 1990-2016: a systematic subnational analysis for the Global Burden of Disease Study 2016. Lancet. 2018; 392(10149):760-775. doi: http://dx.doi.org/10.1016/ S0140-6736(18)31221-2.

10. Moreira JC, Azevedo DB, Gouveia PA, et al. Profile of women with breast cancer. Rev Enferm UFPE on line. 2017;11(6):2264-72. doi: https://doi.org/10.5205/19818963-v11i6a23386p2264-2272-2017.

11. Ministério da Saúde (BR). Diretrizes metodológicas: estudos de avaliação econômica de tecnologias em saúde. Brasília, DF: Ministério da Saúde; 2009. (Série A. Normas e Manuais Técnicos).

12. Guedes JBR, Guerra MR, Alvim MM, et al. Factors associated with adherence and persistence to hormonal therapy in women with breast cancer. Rev Bras Epidemiol. 2017;20(4):636-649. doi: http://dx.doi. org/10.1590/1980-5497201700040007.

13. Brito C, Portela MC, Vasconcellos MTL. Factors associated to persistence with hormonal therapy in women with breast cancer. Rev Saúde Pública. 2014;48(2):284-295. doi: http://dx.doi.org/10.1590/ S0034-8910.2014048004799.

14. Oliveira RS, Menezes JTL, Gonçalves MGL. Adesão à terapia hormonal adjuvante oral em pacientes com câncer de mama. Rev Bras Cancerol. 2012;58(4):593-601.

15. Silva PF, Amorim MHC, Zandonade E, et al. Associação entre variáveis sociodemográficas e estadiamento clínico avançado das neoplasias da mama em hospital de referência no estado do Espírito Santo. Rev Bras Cancerol. 2013;59(3):361-367.

16. Aguiar ADF, Padilha KM, Volpi PTM, et al. Perfil sociodemográfico e clínico das pacientes em tratamento do câncer mamário Rev Inst Ciênc Saúde. 2008;26(2):191-5. 
17. Haddad NC, Carvalho ACA, Novaes CO. Perfil sócio demográfico e de saúde de mulheres submetidas à cirurgia para câncer de mama. Revista HUPE. 2015;14(1):28$35,2015$.

18. Vasconcello RG, Uemura G, Schirmbeck T, et al. Ultrassonografia mamária: aspectos contemporâneos. Com Ciências Saúde. 2011;22(Suppl 1):S129-S140.

19. Rocha ACC. Correlação da classificação BI-RADS ${ }^{\oplus}$ na mamografia e no diagnóstico definitivo em pacientes sintomáticas ou não no Serviço de Mamografia do Hospital da Universidade Federal de Santa Catarina [trabalho de conclusão de curso]. [Santa Catarina]: Universidade Federal de Santa Catarina, Florianópolis; 2006. 50 p.

20. El-Beshbishy H. Hepatoprotective effect of green tea (Camellia sinensis) extract against tamoxifen-induced liver injury in rats. J Biochem Mol Biol. 2005;38(5):563570.

21. Barbosa ARF, Pereira NML, Fook JMSL, et al. O efeito do tamoxifeno no perfil lipídico, hepático e função renal de pacientes portadoras de câncer de mama. PubliCa [Internet]. 2008[acesso 2017 Ago 2];4(2):4147. Disponível em: https://periodicos.ufrn.br/publica/ article/view/93/89.

22. Kaliks RA, Pontes LB, Bognar CLFB, et al. Treatment of breast cancer patients from a public healthcare system in a private center: costs of care for a pilot publicprivate partnership in oncology. Einstein (Sao Paulo). 2013;11(2):216-23. doi: http://dx.doi.org/10.1590/ S1679-45082013000200014.

23. Peregrino AAF, Vianna CMM, Caetano R, et al. Análise de custo-efetividade da idade de início do rastreamento mamográfico. Rev Bras Cancerol. 2010;56(2):187-193.

24. Ministério da Saúde (BR), Secretaria Executiva, Departamento de Economia da Saúde, Investimentos e Desenvolvimento. Núcleos de Economia da Saúde: orientaçóes para implantação. Brasília, DF: Ministério da Saúde; 2012. (Série A. Normas e Manuais Técnicos); (Série gestão e economia da saúde; v. 1) 